\title{
Visual function in patients of a polytrauma rehabilitation center: A descriptive study
}

\author{
Gregory L. Goodrich, PhD; ${ }^{*}$ Jennine Kirby, OD; $^{1}$ Glenn Cockerham, MD; ${ }^{2-3}$ Shanida P. Ingalla, OD; ${ }^{2}$ Henry L. \\ Lew, MD, PhD $^{3-4}$ \\ ${ }^{1}$ Psychology Service and Western Blind Rehabilitation Center and ${ }^{2}$ Ophthalmology Service, Department of Veterans \\ Affairs Palo Alto Health Care System (VAPAHCS), Palo Alto, CA; ${ }^{3}$ Stanford University School of Medicine, Stanford, \\ $C A ;{ }^{4}$ Physical Medicine and Rehabilitation Service, VAPAHCS, Palo Alto, CA
}

\begin{abstract}
Little is known about the visual function deficits associated with polytrauma injury. In this retrospective descriptive study, we examined the records of a clinic established to assess visual function in patients experiencing deployment-related polytrauma. We describe the clinical findings and present a vision examination protocol that may be useful for screening polytrauma patients in other settings. Data from our sample suggested that self-reported vision complaints were common $(74 \%)$ and confirmed that visual impairment occurred in $38 \%$ of all cases. When examining the mechanism of injury, we found that polytrauma due to blast injuries appeared to more than double the risk of visual impairment compared with all other polytrauma causes (i.e., motor vehicle accidents, gunshot and/or shrapnel, assault, falls, or anoxia). The rate of visual impairment in blast-related injury was 52\% compared with $20 \%$ for all other sources of injury. Visual complaints and impairments were common in the polytrauma patients studied. This finding suggests that comprehensive eye examinations should be routinely administered, particularly when the mechanism of injury involves a blast.
\end{abstract}

Key words: adult, blast injury, brain injury, mechanism of injury, ocular refraction, polytrauma, rehabilitation, vision, visual impairment, visual perception.

\section{INTRODUCTION}

Blindness and visual impairment have long been recognized as consequences of war. The eyes encompass only about 0.25 percent of the surface area of the body. Current estimates, however, suggest that injuries to the eyes are 20 to 50 times greater than would be predicted by their relatively small surface area [1-2]. Ocular injuries in World War I constituted between 2.5 and 3.0 percent of all injuries, while in Operations Desert Shield and Storm (1991), they accounted for 13 percent of all injuries [1]. The use of explosive devices has led to an increased number of military personnel with traumatic brain injury (TBI) as a result of combat or related events. TBI may damage the visual pathway, visual cortex, and/or pathways that connect the eyes to cortical visual centers. Other sensory and motor areas, as well as areas governing memory, cognition, motor control, and emotion [3], may also be damaged. Thus, injuries to either the eye or brain may result in vision

Abbreviations: ICD-9 = International Classification of Diseases-9th Edition, logMAR = logarithm of minimum angle of resolution, $\mathrm{MVA}=$ motor vehicle accident, $\mathrm{OEF}=$ Operation Enduring Freedom, OIF $=$ Operation Iraqi Freedom, OPTIC = Optometry Polytrauma Inpatient Clinic, PRC = Polytrauma Rehabilitation Center, SD = standard deviation, $\mathrm{TBI}=$ traumatic brain injury, VA = Department of Veterans Affairs, VAPAHCS = VA Palo Alto Health Care System.

*Address all correspondence to Gregory L. Goodrich, PhD; Psychology Service and Western Blind Rehabilitation Center, VAPAHCS, 3801 Miranda Avenue, Palo Alto, CA 94304; 650-493-5000, ext 64385; fax: 650-852-3239.

Email: Gregory.Goodrich@va.gov

DOI: 10.1682/JRRD.2007.01.0003 
loss or blindness in addition to other functional, emotional, and mental impairments.

Between January 2003 and March 2006, 28 percent of the patients evacuated to Walter Reed Army Medical Center received a diagnosis of TBI and the Defense and Veterans Brain Injury Center classified more than half of these cases as moderate or severe [4]. In a study at the Department of Veterans Affairs (VA) Palo Alto Health Care System (VAPAHCS), 74 percent of 38 combat-related TBI patients in the Polytrauma Rehabilitation Center (PRC) had blast exposure [3]. The relatively high rate of eye injuries may be related to the increased number of head injuries associated with blasts caused by bombs, grenades, rockets, or other explosives as compared with other mechanisms of injury such as gunshots [5-6]. The increased incidence of head and eye injuries has led to continuing efforts to develop better head armor for soldiers [7].

While injuries to the eye and brain have been highlighted in the polytrauma and related literature, little or no attention has been given to the nature of the vision loss. The Veterans Health Administration clinical directive has defined "polytrauma" as injury to the brain concurrently with injury to several other body areas or organ systems that results in physical, cognitive, and psychosocial impairments. ${ }^{*}$ The lack of understanding of vision loss and its possible interaction with brain injury may interfere with effective rehabilitation. A similar situation exists in the stroke and TBI literature. Zihl [8] and Biehl et al. [2] have noted that central visual and oculomotor disorders occur in 20 to 40 percent of neurological rehabilitation center patients, and Gianutsos reported that about half of head trauma patients exhibit visual system disorders that have not been assessed despite previous hospital treatment [9]. Kerkhoff suggested that two factors explain the lack of eye examinations and treatment: limited financial resources in healthcare and the belief that central visual disorders do not require treatment, since they do not adversely affect outcome [10]. Kerkhoff questioned the validity of that belief and presented evidence that although selected training activities do not restore normal function, they do increase independence and perceived quality of life [10].

\footnotetext{
*Department of Veterans Affairs, Veterans Health Administration. Polytrauma rehabilitation centers. Washington (DC): Veterans Health Administration; 2005.
}

Visual impairments adversely affect the subjective quality of life of institutionalized [10] and noninstitutionalized [11-12] individuals. On a functional level, visual impairments reduce the individual's ability to perform such common tasks as reading, activities of daily living, facial recognition, and travel. Visual impairments are also associated with increased length of hospitalization [13], risk of falls and hip fracture [14-15], and mortality [1617]. Experience in the VA Blind Rehabilitation Service has shown that prescribing optical devices and specialized rehabilitation interventions restores independent function in cases of severe visual impairment, including blindness.

The brain is particularly susceptible to high-impact injuries and, as Lew noted [18], compensation strategies for rehabilitation rely heavily on the brain. Vision can play an important role in polytrauma rehabilitation since walking, reading, face recognition, orientation, shopping, meal preparation, grooming, and a myriad of other daily activities derive most of their sensory input from the visual system. An estimated 70 percent of all human sensory processing is affected by information from the two eyes [19]. Unrecognized or uncorrected refractive errors, photosensitivity, or more severe visual and/or perceptual impairment in polytrauma patients are likely to negatively affect rehabilitation efforts. Uncorrected refractive errors, for example, may reduce performance on penand-paper neuropsychological tests. Progress in the rehabilitation of activities of daily living may be impeded if the patient cannot clearly see labels, read directions, or recognize common objects. More severe visual impairments, such as hemianopsia or visual neglect, may hinder physical therapy for walking or wheelchair use and increase balance problems and fall risks.

The severe combination of disabilities presented by polytrauma patients is an unprecedented challenge for rehabilitation facilities. This challenge has led some to propose that, while acute emergency-based medical care is well described, it may not be an adequate model for treating this unique population in rehabilitation settings, particularly when patients have blast-related injuries. Scott and colleagues proposed mechanism of injury as the preferred model for comprehensively assessing and treating blast-related polytrauma cases [20]. A major advantage of this model appears to be its focus on the typical sequelae given the mechanism of injury, which increases the likelihood that related conditions will be detected. Undetected visual impairment, for example, can be masked by symptoms associated with problems in cognition and 
communication, posttraumatic stress disorder, or other conditions common in polytrauma patients.

The current study quantified and analyzed the results of comprehensive vision examinations conducted on a cohort of polytrauma patients who were deployed in conjunction with Operation Iraqi Freedom (OIF) or Operation Enduring Freedom (OEF). These patients' injuries occurred either during combat or deployment-related activities. For example, all injuries from a blast event were combat-related, as were gunshot and/or shrapnel wounds. Motor vehicle accidents (MVAs) were not always combat-related. All injuries due to falls were non-combat-related.

For this article, we defined "vision deficits" broadly to encompass conditions such as uncorrected refractive error and photosensitivity as well as more significant vision losses such as visual impairment and blindness. We also report on patients who had visual perceptual deficits (e.g., visual neglect or inattention) and/or difficulty reading (e.g., inability to comprehend at a fifth-grade level, scan or track lines of text, or read fluently as rated by the examining optometrist) even though their visual acuity may have been normal or near normal. Of note, the vision examinations revealed conditions that likely existed before injury (e.g., refractive error) in addition to conditions that appeared to be sequelae of the events that resulted in the polytrauma injury. However, drawing cause-and-effect conclusions is beyond the scope of the current article. Rather, our focus is on elucidating those visual characteristics of a cohort of polytrauma patients that may limit or impede rehabilitation.

\section{METHODS}

We derived data for the current study from a record review of patients seen in the Optometry Polytrauma Inpatient Clinic (OPTIC) at VAPAHCS between its opening in December 2004 and November 2006. The OPTIC was established specifically as a referral point for eye examinations for patients in the PRC and for refractive corrections and other appropriate remediation. In addition, the OPTIC provided information on the visual status of polytrauma patients to members of their PRC treatment team and referred patients to other services when appropriate. A specific eye complaint was unnecessary for referral, and the clinic attempted to see all veterans and active-duty personnel admitted to the PRC. All subject data collection methods complied with regulations regarding the treatment of human subjects in research. The Stanford University/VAPAHCS Institutional Review Board approved the study.

The OPTIC examination protocol is outlined in the Appendix (available online only at http:// www.rehab.research.va.gov/). It includes self-report questions about vision status before and after the injury, clinical measurements of distance and near visual acuity, visual fields, binocular vision status, and other vision measures. Patients were usually referred for OPTIC examinations soon after arriving at the PRC. Patients were examined at bedside if they could not sit up or had insufficient mobility to travel to the eye clinic for a standard examination. To allow vision examinations of even severely impaired patients, the eye examination protocol used the Feinbloom chart (Optelec US Inc, Vista, California) for distance visual acuity and the Lighthouse Letter Chart (Optelec US Inc) for near visual acuity testing. In addition, Teller Acuity Cards (Optelec US Inc) were used as needed for very low-functioning patients who were limited in cognitive ability and/or ability to verbally respond to tests.

Visual fields conducted at bedside were screened with confrontation fields. If the confrontation field indicated a possible field loss, a visual field was performed with either a hand-held arc perimeter, or if the patient could travel, a Goldmann perimeter (no longer commercially available). Reading was assessed with paragraphlength, fifth-grade equivalent, $1.0 \mathrm{M}$ print materials with correction if needed. The clinician used a three-point scale (i.e., smooth, hesitating, no facility) to rate the subject's ability to read aloud. Reading speed and the ability to understand or remember the content of the paragraph were tested with five questions about the content. Comprehension was also rated on a three-point scale: complete (five of five questions answered correctly), basic (two to four questions answered correctly), or does not comprehend (zero or one question answered correctly).

Accommodative amplitude was measured in each eye with a push-up/pull-away technique. Near point of convergence was measured with a confrontation near target. Fixation stability on a near target was assessed for steadiness, and any nystagmus was noted. Saccadic eye movements were assessed for accuracy and speed of eye movements between targets. Pursuit eye movements were assessed for accuracy and smoothness. Extraocular muscles were assessed for range of motion and equality between the eyes.

Binocular vision function was assessed with cover tests in primary gaze at distance and at near to detect potential convergence, divergence, or strabismus problems. 
Stereo testing with the Randot test (Bernell VTP, Misawaka, Indiana) was used to assess fine binocular function. Color vision was screened with Ishihara plates (Bernell VTP) to detect congenital and/or pathological color vision dysfunction. Pupils were assessed with the swinging flashlight test to exclude optic nerve involvement. Damage to cranial nerve II was assessed by visual acuity, color vision, pupil testing, and optic atrophy. Enucleated eyes were considered to have damage to cranial nerve II. The status of cranial nerves III, IV, and VI was assessed by using extraocular muscles and cover test.

Visual perceptual and spatial deficits were assessed in the speech pathology examination, and data for the current study were taken from the speech pathologists' entries in the patients' medical charts. Eye examinations for easily fatigued patients were broken up over several days.

Findings outside the normal ranges for saccades, pursuits, fixation and/or nystagmus, accommodation, and convergence were considered dysfunctions. Visual impairment was defined based on the International Classification of Diseases-9th Edition (ICD-9) classification; however, some ICD-9 levels were combined to reflect VA eligibility for vision rehabilitation service. ${ }^{*}$ Normal and near-normal levels were combined into a single normal/near-normal level (visual acuity between 20/20 and 20/63). Levels of moderate, severe, profound, and near-total visual impairment were combined into a single visual impairment category (visual acuity ranging from 20/80 to 20/1,000). Hemianopsia was classified under ICD-9 as a severe visual impairment comparable with a visual field of $\leq 20^{\circ}$. Blindness was classified as a visual acuity of $<20 / 1,000$ or bilateral enucleation.

\section{RESULTS}

The current study reports on 50 patients examined in the OPTIC. This group represents 70 percent of all the patients admitted to the PRC from December 2004 (when the OPTIC opened) to November 2006. The subjects lost to this study included those who were transferred or discharged to another facility before an examination could be scheduled or completed, those who could not respond to the examination (i.e., emerging consciousness patients), or

* OEF and OIF veterans classified as visually impaired or blind are eligible for services provided by the VA Blind Rehabilitation Service. those who were not referred during the start-up phase of the clinic. The mean age of subjects examined was 28.1 years (median 26 years), with a range of 19-56 years; 5 subjects were female (10\%) and 45 were male (90\%).

All subjects had experienced a TBI, as diagnosed and documented in their medical records by the Palo Alto Defense and Veterans Brain Injury Center; however, TBI was not an inclusion criterion for this study. For this article, we categorized as "blast" injuries all injuries from improvised explosive devices, rocket-propelled grenades, mortars, or other explosive causes. Blast injuries accounted for half of all injuries. MVAs not involving a blast were the second most frequent source of injury (26\%), followed by assault and falls (8\% each), gunshot and/or shrapnel wounds (4\%), and anoxia (4\%). Most injuries (59\%, $n=$ $29)$ occurred in combat, while the remainder $(41 \%, n=21)$ occurred in non-combat-related assaults, MVAs, or falls. More than half (56\%) of the head injuries were nonpenetrating. Blast-related injuries produced penetrating injuries to the head in 44 percent of occurrences, while all other injury causes produced penetrating injuries in 16 percent of occurrences. A total of 74 percent of subjects complained about vision. These subjects reported one or more of the following: blurred distance vision, sensitivity to light, missing a part of their vision, bumping into objects or walls, blurred near-reading vision, and inability to comfortably read continuous text. In addition, 6 percent of subjects were unresponsive to the questions and 20 percent reported no vision difficulties.

The average best-corrected visual acuity for right eyes (20/34, logarithm of minimum angle of resolution [log$\mathrm{MAR}]=0.236$, standard deviation $[\mathrm{SD}]=0.419$ ) and left eyes $(20 / 36, \operatorname{logMAR}=0.264, \mathrm{SD}=0.432)$ was similar to the mean binocular visual acuity of 20/34 (logMAR = 0.230 , SD $=0.412$ ). Sixty-four percent of the subjects had a refractive error and required correction. Many did not have their glasses or contacts with them upon admission because these items were lost or misplaced during transit and treatment from the battlefield to the PRC.

Most subjects had normal visual fields; however, visual field defects were common (Table 1). In some cases, visual field assessments could not be performed because the eye was enucleated or the patients' visual acuity was insufficient for testing; these cases were classified as "not applicable." In a small number of cases, subjects were unable to verbally or otherwise respond to the field stimulus in a manner that could be interpreted; these cases were classified as "unknown/untested." 
A total of 18 percent of subjects had one eye enucleated, and 8 percent were blind in both eyes. In these blind eyes, both eyes were either enucleated or had light perception only. All cases of total blindness were associated with a blastinduced injury. Blindness or legal blindness was present in seven cases (14\%). Blasts accounted for three of these cases (6\%), gunshot wounds for two (4\%), and falls or assault for one each (2\%). Visual impairments (visual acuity between 20/63 and 20/100) occurred in five cases (10\%), with blasts accounting for four (8\%) and an MVA for one (2\%).

Table 2 presents the number and percentage of subjects who were not classified as visually impaired but sustained injury to the eye, orbit, and/or cranial nerves. For simplicity and to reflect the difficulty in assessing specific cranial nerve injury in this population, we sepa-

Table 1.

Visual field characteristics of 50 polytrauma subjects. Data shown as No. (\%) of subjects.

\begin{tabular}{lcc}
\hline \multicolumn{1}{c}{ Characteristic } & Right Eye & Left Eye \\
\hline Normal or Near Normal & $30(60)$ & $31(62)$ \\
Not Applicable (enucleation or no & $8(16)$ & $5(10)$ \\
$\quad$ light perception) & $3(6)$ & $1(2)$ \\
Not Measurable & $3(6)$ & $3(6)$ \\
Field Constricted & $0(0)$ & $2(4)$ \\
Paracentral Scotoma & $3(6)$ & $4(8)$ \\
Left Hemianopsia with Macular & & \\
$\quad$ Sparing & $0(0)$ & $1(2)$ \\
Right Hemianopsia with Macular & & \\
$\quad$ Sparing & $0(0)$ & $1(2)$ \\
Left Hemianopsia with Macular & & \\
$\quad$ Splitting & $1(2)$ & $0(0)$ \\
Left Inferior Quadranopsia & $1(2)$ & $0(0)$ \\
Left Superior Quadranopsia & $1(2)$ & $1(2)$ \\
Superior Altitudinal Defect &
\end{tabular}

Table 2.

No. (\%) of subjects with damage to eye, orbit, cranial nerve II, and/or other cranial nerve (III, IV, or VI) among all nonvisually impaired subjects $(n=31)$.

\begin{tabular}{llll}
\hline \multicolumn{1}{c}{ Eye } & $\begin{array}{c}\text { Eye or } \\
\text { Orbit }\end{array}$ & $\begin{array}{c}\text { Cranial } \\
\text { Nerve II }\end{array}$ & $\begin{array}{c}\text { Other } \\
\text { Cranial } \\
\text { Nerve }\end{array}$ \\
\hline Left & $5(16.1)$ & $1(3.2)$ & $2(6.5)$ \\
Unknown/Untested & $0(0)$ & $1(3.2)$ & $2(6.5)$ \\
Right & $3(9.7)$ & $2(6.5)$ & $3(9.7)$ \\
Unknown/Untested & $0(0)$ & $2(6.5)$ & $2(6.5)$ \\
Both & $0(0)$ & $0(0)$ & $0(0)$ \\
\hline \hline
\end{tabular}

rated the data for cranial nerve II and aggregated the data for cranial nerves III, IV, and VI. Table 3 presents the same data for subjects who were classified as visually impaired. Overall, those patients whose injury resulted in a visual impairment were more likely to have damage to the eye, orbit, and/or cranial nerves. Table 4 presents the number and percentage of subjects whose injuries were non-blast-related but who sustained damage to the eye, orbit, and/or cranial nerves. Table 5 presents the same data for those subjects whose injuries were blast-related.

Table 3.

No. (\%) of subjects with damage to eye, orbit, cranial nerve II, and/or other cranial nerve (III, IV, or VI) among all visually impaired subjects $(n=19)$.

\begin{tabular}{llll}
\hline \multicolumn{1}{c}{ Eye } & $\begin{array}{c}\text { Eye or } \\
\text { Orbit }\end{array}$ & $\begin{array}{c}\text { Cranial } \\
\text { Nerve II }\end{array}$ & $\begin{array}{c}\text { Other } \\
\text { Cranial } \\
\text { Nerve }\end{array}$ \\
\hline Left & $2(10.5)$ & $5(26.3)$ & $3(15.8)$ \\
Unknown/Untested & $0(0)$ & $2(10.5)$ & $2(10.5)$ \\
Right & $3(15.8)$ & $3(15.8)$ & $5(26.3)$ \\
Unknown/Untested & $0(0)$ & $7(36.8)$ & $5(26.3)$ \\
Both & $4(21.1)$ & $0(0)$ & $0(0)$ \\
\hline
\end{tabular}

Table 4.

No. (\%) of subjects with damage to eye, orbit, cranial nerve II, and/or other cranial nerve (III, IV, or VI) among all non-blast-related polytrauma subjects $(n=25)$.

\begin{tabular}{lccc}
\hline \multicolumn{1}{c}{ Eye } & $\begin{array}{c}\text { Eye or } \\
\text { Orbit }\end{array}$ & $\begin{array}{c}\text { Cranial } \\
\text { Nerve II }\end{array}$ & $\begin{array}{c}\text { Other } \\
\text { Cranial } \\
\text { Nerve }\end{array}$ \\
\hline Left & $2(8)$ & $1(4)$ & $2(8)$ \\
Unknown/Untested & $0(0)$ & $0(0)$ & $0(0)$ \\
Right & $2(8)$ & $2(8)$ & $3(12)$ \\
Unknown/Untested & $0(0)$ & $0(0)$ & $1(4)$ \\
Both & $0(0)$ & $0(0)$ & $0(0)$ \\
\hline \hline
\end{tabular}

Table 5.

No. (\%) of subjects with damage to eye, orbit, cranial nerve II, and/or other cranial nerve (III, IV, or VI) among all blast-related polytrauma subjects $(n=25)$.

\begin{tabular}{llll}
\hline \multicolumn{1}{c}{ Eye } & $\begin{array}{c}\text { Eye or } \\
\text { Orbit }\end{array}$ & $\begin{array}{c}\text { Cranial } \\
\text { Nerve II }\end{array}$ & $\begin{array}{c}\text { Other } \\
\text { Cranial } \\
\text { Nerve }\end{array}$ \\
\hline Left & $5(20)$ & $6(24)$ & $4(16)$ \\
Unknown/Untested & $0(0)$ & $3(12)$ & $4(16)$ \\
Right & $4(16)$ & $2(8)$ & $4(16)$ \\
Unknown/Untested & $0(0)$ & $9(36)$ & $6(24)$ \\
Both & $4(16)$ & $0(0)$ & $0(0)$ \\
\hline \hline
\end{tabular}


Blast-related injuries were more frequently associated with damage to the eye, orbit, and/or cranial nerves.

Binocular, perceptual, and reading difficulties were common in subjects regardless of the mechanism of injury (Table 6). Accommodation, convergence, and spatial deficits occurred in 20 percent or more of all subjects and within each mechanism-of-injury category. Reading deficits were also equally represented overall and within each category but at a much higher rate $(\sim 60 \%)$ than other deficits. Best-corrected visual acuity was correlated with reading deficits; however, the correlation was modest $(r=$ $0.419, p<0.001$ ) and accounted for less than 18 percent of the variance. Blast-related injuries and non-blast-related injuries were associated with similar rates of binocular or perceptual dysfunction. Non-blast-related injuries, however, were associated with somewhat higher rates of convergence and pursuit and/or saccade dysfunction, fixation and/or nystagmus, and diplopia.

\section{DISCUSSION}

We assessed the visual characteristics of OIF/OEF troops admitted to the VAPAHCS PRC. The visual examination was designed to uncover a wide range of diagnoses that were either preexisting or related to the injuries that resulted in the patient's admission to the PRC. In addition to the visual examination, the study gathered self-report data from each patient about his or her current visual function as well as patient-record data relating to the presence or absence of TBI and visual perceptual and/or spatial disorders. The data generated by this study suggest that if eye examinations only assessed visual acuity, one might well conclude that further eye examinations were unnecessary unless warranted by the patient's injuries. The data indicated that the median best-corrected visual acuity in the polytrauma population was $20 / 20$, which is classified as "normal." However, the study also found that 74 percent of patients reported vision-related complaints and 64 percent had refractive errors that required optical correction. Refractive errors were probably unrelated to the injuries leading to these patients' admission to the PRC. Often, the patients' optical corrections, such as glasses or contact lenses, had been misplaced or lost before the examination. In such cases, appropriate corrections were prescribed because they are necessary for individuals to perform routine tasks such as reading; recognizing friends, family, or staff; watching television; and other daily tasks. The absence of corrective lenses can result in myopic patients, who experience difficulty seeing signs, watching television, or recognizing distant people or objects, or in hyperopic patients, who may have difficulty reading or performing near tasks, including rehabilitation-related assessments. Combined with a TBI, the need for refractive correction can lead to unnecessarily increased difficulty and frustration for patients trying to accomplish even simple tasks.

More significant visual deficits were also common. About 24 percent of subjects had visual field deficits, while 38 percent of subjects sustained vision loss that ranged from moderate to total blindness as a result of visual acuity, visual field loss, and/or bilateral enucleation. Blast-related injuries were associated with more cases of vision loss (52\%) than were other mechanisms of injury (20\%). These patients' degree of vision loss made them eligible for VA Blind Rehabilitation services

Table 6.

No. (\%) of binocular, perceptual, or reading problems for all subjects and for subjects grouped by mechanism of injury (blast vs nonblast). Totally blind subjects $(n=4)$, all with blast-related injuries, were excluded.

\begin{tabular}{lccc}
\hline \multicolumn{1}{c}{ Problem } & $\begin{array}{c}\text { All Subjects } \\
(\boldsymbol{n}=\mathbf{4 6 )}\end{array}$ & $\begin{array}{c}\text { Blast } \\
(\boldsymbol{n}=\mathbf{2 1})\end{array}$ & $\begin{array}{c}\text { Nonblast } \\
(\boldsymbol{n}=\mathbf{2 5})\end{array}$ \\
\hline Accommodation Dysfunction & $10(21.7)$ & $5(23.8)$ & $5(20.0)$ \\
Convergence Dysfunction & $14(30.4)$ & $5(23.8)$ & $9(36.0)$ \\
Pursuit/Saccade Dysfunction & $9(19.6)$ & $1(4.8)$ & $8(32.0)$ \\
Fixation/Nystagmus & $1(2.2)$ & $0(0)$ & $1(4.0)$ \\
Diplopia & $3(6.5)$ & $0(0)$ & $3(12.0)$ \\
Suppression & $7(15.2)$ & $3(14.3)$ & $4(16.0)$ \\
Neglect & $4(8.7)$ & $2(9.5)$ & $2(8.0)$ \\
Visual Spatial Deficit & $14(30.4)$ & $6(28.6)$ & $8(32.0)$ \\
Reading & $28(60.9)$ & $13(61.9)$ & $15(60.0)$ \\
\hline \hline
\end{tabular}


and many were referred for these services. However, physical or cognitive injuries precluded an immediate referral in some cases. In other cases, the patients wanted to return home before receiving additional rehabilitation.

When we compared the incidence of damage to the eye, orbit, and/or cranial nerves (II, III, IV, or VI) between subjects who were and were not classified as visually impaired, we found that both groups had similar damage to the eye and orbit. The group classified as visually impaired, however, showed somewhat higher rates of cranial nerve damage. When we examined damage to the cranial nerves by mechanism of injury (blast versus all other causes), we found that blasts were associated with higher rates of damage to the eye, orbit, and/or cranial nerves. Damage to the cranial nerves can result in reduced visual acuity, decreased or lost pupillary response, strabismus, or other oculomotor disorders that interfere with binocular vision and/or the ability to effectively fixate and track moving objects.

For all subjects, the incidence of binocular dysfunction (i.e., problems with accommodation or convergence), pursuit and/or saccade deficits, and visual spatial deficits ranged between 20 and 30 percent. These deficits showed no consistent differences by mechanism of injury. As measured by reading ability and comprehension of a fifth-gradelevel paragraph, most subjects' reading was impaired (rated as basic comprehension or no comprehension); however, the mechanism of injury did not differentiate reading ability. Poor visual acuity was correlated with poorer reading performance. This modest correlation, however, left 80 percent of the variance unaccounted for, which suggests that factors other than reduced visual acuity were equally or more important in producing reading deficits. Visual field restrictions may be one such factor. Also, given that all subjects were diagnosed with a TBI, the reduced reading ability might have been the result of cognitive, perceptual, attentional, or other factors. The current study lacks information about the population's preinjury reading level and, although we consider it unlikely that most of these individuals could not read at a fifth-grade level before injury, we cannot rule out prior poor reading ability as a contributing factor.

Visual deficits, including severe visual impairments, appear to be common in polytrauma patients. The current study strongly suggests that damage to the optical and visual perceptual system is a major contributor to the constellation of deficits shown by polytrauma patients. These results appear to be robust. However, one should note that the population studied was relatively small. Also, because the Palo Alto PRC is the only one of the four PRCs to have blind rehabilitation, ophthalmology, and optometry services, there may have been a bias in referring patients with visual impairments to Palo Alto. While we have no evidence of such bias, we cannot rule it out. Even if such referral bias did exist, we consider it unlikely that it would be sufficient to negate the findings of this study.

Among the constellation of injuries experienced by polytrauma patients, vision-related loss is prevalent, and if unaddressed, likely to negatively impact rehabilitation. The current study results should encourage additional research on the extent and nature of vision loss and its rehabilitation in the polytrauma population. The role of mechanism of injury in defining assessment and treatment strategies for individuals exposed to improvised explosive devices and other high-powered blasts should be further explored. Such future studies can build on the current study by including a larger sample size and improving current assessment techniques. The assessment of cranial nerve damage in particular would benefit from a more sophisticated strategy than was available to us.

\section{CONCLUSIONS}

The current study found evidence of frequent and severe visual impairments in a population of polytrauma patients. The extent and severity of vision-related injury and deficit suggest that all polytrauma patients should have routine comprehensive vision examinations. The relationship found between vision loss or dysfunction and blast events supports the concept that the "mechanism-of-injury" model is valuable in polytrauma assessment and treatment settings. The current findings are consistent with the need for additional studies on vision and visual perceptual loss in polytrauma patients. Such studies should not be limited to patients with overt blastrelated injuries. Given the magnitude of the effect of blasts on the visual system, studying troops exposed to blasts, even if minor or with no resulting physical damage, would seem prudent. Such study would be particularly relevant for those troops exposed to a blast who report any level of visual difficulty, such as decreased reading ability, reduced reading duration, inability to track printed materials, or photosensitivity. The Palo Alto Polytrauma Network Site clinic has undertaken a study incorporating a visual screen, and the results of that study will be reported in another publication. 


\section{ACKNOWLEDGMENTS}

We wish to thank Ms. Rose Salerno, RN, for her assistance in documenting the number of polytrauma admissions during the reporting period. We also wish to thank the staff of the VAPAHCS PRC for their cooperation and dedication. Thanks also to Drs. Karen Brahm and Mimi Shen for their critical and comprehensive review of the manuscript. Deserving of special recognition are the patients of the PRC, whose courage and determination in the face of seemingly overwhelming injury inspire all who meet them.

This material was based on work supported in part by the VA (grant B3288R to Henry Lew, MD, PhD).

The authors have declared that no competing interests exist.

\section{REFERENCES}

1. Wong TY, Seet MB, Ang CL. Eye injuries in twentieth century warfare: A historical perspective. Surv Ophthalmol. 1997;41(6):433-59. [PMID: 9220567]

2. Biehl JW, Valdez J, Hemady RK, Steidl SM, Bourke DL. Penetrating eye injury in war. Mil Med. 1999;164(11): 780-84. [PMID: 10578588]

3. Lew HL, Poole JH, Guillory SB, Salerno RM, Leskin G, Sigford B. Persistent problems after traumatic brain injury: The need for long-term follow-up and coordinated care. J Rehabil Res Dev. 2006;43(2):vii-x. [PMID: 16847779]

4. Defense and Veterans Brain Injury Center [homepage on the Internet]. Washington (DC): Defense and Veterans Head Injury Program; c2007 [updated 2006; cited 2007 Oct 17]. Available from: http://www.dvbic.org

5. Peleg K, Aharonson-Daniel L, Stein M, Michaelson M, Kluge Y, Simo D, Noji EK, Israeli Trauma Group. Gunshot and explosion injuries: Characteristics, outcomes, and implications for care of terror-related injuries in Israel. Ann Surg. 2004;239(3):311-18. [PMID: 15075646$]$

6. Nguyen QD, Kruger EF, Kim AJ, Lashkari MH, Lashkari K. Combat eye trauma: Intraocular foreign body injuries during the Iran-Iraq war (1980-1988). Int Ophthalmol Clin. 2002;42(3):167-77. [PMID: 12131593]

7. Ward TP. The development of eye armor for the American infantryman. Ophthalmol Clin N Am. 1999;12(3):421-34.

8. Zihl J. Cerebral disturbances of elementary visual functions. In: Brown JW, editor. Neuropsychology of visual perception. Hillsdale (NJ): Lawrence Erlbaum Associates; 1989. p. 35-58.

9. Gianutsos R. Vision rehabilitation following acquired brain injury. In: Gentile M, editor. Functional visual behavior: A therapist's guide to evaluation and treatment options. Bethesda (MD): American Occupational Therapy Association; 1997. p. 267-94.

10. Kerkhoff G. Neurovisual rehabilitation: Recent developments and future directions. J Neurol Neurosurg Psychiatry. 2000;68(6):691-706. [PMID: 10811691]

11. Brezin AP, Lafuma A, Fagnani F, Mesbah M, Berdeaux G. Prevalence and burden of self-reported blindness and low vision for individuals living in institutions: A nationwide survey. Health Qual Life Outcomes. 2005;3:27. [PMID: 15847703]

12. Hassell JB, Lamoureux EL, Keeffe JE. Impact of age related macular degeneration on quality of life. Brit J Ophthal. 2006;90(5):593-96. [PMID: 16622089]

13. Stelmack JA, Szlyk JP, Stelmack TR, Demers-Turco P, Williams RT, Moran D, Massof RW. Measuring outcomes of vision rehabilitation with the Veterans Affairs Low Vision Visual Functioning Questionnaire. Invest Ophthal Vis Sci. 2006;47(8):3253-61. [PMID: 16877389]

14. Morse AR, Yatzkan E, Berberich B, Arons RR. Acute care hospital utilization by patients with visual impairment. Arch Ophthalmol. 1999;117(7):943-49. [PMID: 10408461]

15. Ivers RQ, Norton R, Cumming RG, Butler M, Campbell AJ. Visual impairment and risk of hip fracture. Am J Epidemiol. 2000;152(7):633-39. [PMID: 11032158]

16. Jack CI, Smith T, Neoh C, Lye M, McGalliard JN. Prevalence of low vision in elderly patients admitted to an acute geriatric unit in Liverpool: Elderly people who fall are more likely to have low vision. Gerontology. 1995;41(5): 280-85. [PMID: 8537012]

17. Lee DJ, Gomez-Marin O, Lam BL, Zheng DD, Caban A. Visual impairment and morbidity in community-residing adults: The national health interview survey 1986-1996. Ophthalmic Epidemiol. 2005;12(1):13-17.

[PMID: 15848916]

18. Lew HL. Rehabilitation needs of an increasing population of patients: Traumatic brain injury, polytrauma, and blastrelated injuries. J Rehabil Res Dev. 2005;42(4):xiii-xvi. [PMID: 16320135]

19. Jacobs JM, Hammerman-Rozenberg R, Maaravi Y, Cohen A, Stessman J. The impact of visual impairment on health, function and mortality. Aging Clin Exp Res. 2005;17(4): 281-86. [PMID: 16285193]

20. Scott SG, Belanger HG, Vanderploeg RD, Massengale J, Scholten J. Mechanism-of-injury approach to evaluating patients with blast-related polytrauma. J Am Osteopath Assoc. 2006;106(5):265-70. [PMID: 16717367]

Submitted for publication January 8, 2007. Accepted in revised form July 12, 2007. 